Retrospective Study

\title{
The Incidence and Risk Factors for Lumbar or Sciatic Scoliosis in Lumbar Disc Herniation and the Outcomes after Percutaneous Endoscopic Discectomy
}

Ranhee Kim, BS ${ }^{1}$, Rae Hyung Kim, BS ${ }^{1}$, Chi Heon Kim, MD, PhD 2,3,4, Yunhee Choi, PhD ${ }^{5}$, Hyun Sook Hong, MS ${ }^{5}$, Sung Bae Park, MD ${ }^{2,3,4,6}$, Seung Heon Yang, MD ${ }^{2,3}$, Sung-Mi Kim, $\mathrm{RN}^{2}$, and Chun Kee Chung, MD, PhD 2,3,4,5

From: ${ }^{1}$ Department of Medicine, Seoul National University College of Medicine; ${ }^{2}$ Department of Neurosurgery, Seoul National University College of Medicine; 3Department of Neurosurgery, Seoul National University Hospital, Seoul, Korea; ${ }^{4}$ Clinical Research Institute, Seoul National University Hospital,

Seoul, Korea; ${ }^{5}$ Medical Research Collaborating Center, Seoul National University College of Medicine, Seoul,

Korea; Department of Brain and

Cognitive Sciences, Seoul National University College of Natural Sciences, Seoul, Republic of Korea; ${ }^{6}$ Department of Neurosurgery, Seoul National University Boramae Hospital, Seoul,

Korea

Address Correspondence: Chi Heon Kim, MD, PhD Department of Neurosurgery Seoul National University College of Medicine

101 Daehak-Ro, Jongno-gu, Korea E-mail: chiheon1@snu.ac.kr

This work was supported by Grant No. 03-2013-0330 from the Seoul National University Hospital Research

Fund. The authors appreciate the statistical advice from the Medical Research Collaborating Center at the Seoul National University Hospital and the Seoul National University College of Medicine. The senior author (CHK) is a consultant of Richard Wolf $\mathrm{GmbH}$. There is no grant from Richard Wolf GmbH. The other authors declare no conflict of interest concerning the materials or methods used in this study or the findings described in this paper.

Manuscript received: 04-19-2015 Accepted for publication: 05-18-2015

Free full manuscript: www.painphysicianjournal.com
Background: Some patients with lumbar herniated intervertebral disc disease (HIVD) suffer from both pain and lateral shift or trunk list. In addition to pain, patients have concerns regarding whether trunk list is reversible. Surgical treatment is performed when pain is intractable to conservative management, but a reversal of trunk list is an incidental outcome. Percutaneous lumbar endoscopic discectomy (PELD) is one of the surgical treatment options for lumbar HIVD, but no results concerning its effect on trunk list have been reported.

Objectives: The objectives of the present study were to determine the incidence of, and risk factors for, trunk list scoliosis or lateral shift and to report the outcomes of trunk list after PELD.

Study Design: Retrospective case study. IRB No. H 1111-025-384

Setting; University medical Center, Seoul, Korea.

Methods: We selected 164 patients who were less than 60 years old, complained of unilateral leg pain, and underwent PELD. We measured the maximum trunk shift from the central sacral vertical line (CSVL-max) on preoperative whole spine radiographs and classified trunk list as CSVL-max $\geq 10 \mathrm{~mm}$. CSVL-max was measured on serial radiographs taken at one, 3,6 , and 12 months postoperatively in patients with trunk list.

Results: Twenty-nine patients (17.9\%) had trunk list (M:F=10:19; mean age, $37.1 \pm 11.24$ years). Female gender (OR 4.28; 95\% Cl, $1.49-12.3$ ) and HIVD at L4-5 (OR 5.6; $95 \% \mathrm{Cl}$, 1.8 - 16.7) were risk factors for trunk list. Trunk list was normalized (CSVL-max $<10 \mathrm{~mm}$ ) in 15 (52\%) patients after PELD, and the median time for normalization was $3-6$ months. Prognostic factors for the recovery of trunk list were not identified.

Limitations: Selection bias should be considered in interpreting these results.

Conclusion: Trunk list, scoliosis or lateral shift, was observed in $18 \%$ of the patients at the time of surgery. Female gender and L4-5 disc herniation were risk factors for trunk list. Trunk list was reversible in more than $50 \%$ of patients within 6 months of PELD.

Key words: Lumbar, scoliosis, lumbar/sciatic, trunk list, endoscope, disc, discectomy, sciatica, spine, percutaneous endoscopic discectomy

Pain Physician 2015; 18:555-564 


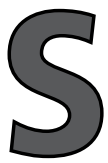
ciatic scoliosis or lateral shift is a term that is used to define a condition in which there is a lateral and forward tilt of the trunk secondary to irritation of the nerve root $(1,2)$. This condition has been identified by a variety of terms in the literature, including sciatic scoliosis $(2,3)$, scoliotic list (4), sciatic scoliotic list $(1,4,5)$, lumbosacral list (6), and trunk list (7-9). Although the terminology varies, all of the terms refer to a deviated and tilted trunk (9). Patients with trunk list are usually between 20 and 50 years of age, in good health, and often very muscular and vigorous; it is not typically observed in older people (2). The appearance of the patients is quite characteristic: they sit, stand, and walk with their trunk shifted laterally and forward (2). Though the etiologies of trunk list, scoliois or later shift, are various, lumbar herniated intervertebral disc disease (HIVD) is a common cause $(1,2,9)$.

In addition to pain, patients often experience concern regarding when and how the trunk list can be reversed. There is evidence that the curvature of trunk list is not due to a structural cause and is reversible with the improvement of pain $(1,2,4,7,10)$. Data after conservative treatment and open discectomy are available in the literature $(1,3,4,7,9,11)$. Percutaneous lumbar endoscopic discectomy (PELD) is a minimally invasive surgical technique that is used for lumbar discectomy (12-14), but no results on its effect on trunk list have been reported. The objectives of the present study were to understand the incidence and risk factors for trunk list and to report the outcomes of trunk list after PELD.

\section{Methods}

A retrospective review was conducted on patients who underwent PELD for medically intractable pain that was present for at least 2 months (12-15). The study received approval from the institutional review board ( $H$ 1111-025-384). From these patients, we selected 220 patients who were younger than 60 years old and who had dominant unilateral leg pain between Sept. 2009 and Dec. 2013. Preoperative whole-spine radiographs were performed on 164 patients using 36-inch-long digital lateral radiographic films, with fists-on-clavicle position and with the hips and knees fully extended (16). We measured trunk shift from the midline on the preoperative whole spine radiographs and classified trunk list as greater than a $10 \mathrm{~mm}$-shift from the midline (Fig. 1) (17). Nearly all patients in the present study underwent epidural spinal injections several times because of regulation of the national health insurance system $(13,18)$.

All patients filled out questionnaires for the Korean version of the Oswestry Disability Index (K-ODI, /45) (19) and visual analogue pain scores (VAS) for the back (VAS-back, /10) and leg (VAS-leg, /10). Patients were encouraged to ambulate on the day of surgery and were discharged the following day. The patients were scheduled to visit the outpatient clinic at one, 3, 6, and 12 months post-operatively, at which time the whole spine radiographs and questionnaires were repeated.

\section{Radiological Measurement}

Patients were asked to stand up and look straight ahead during the whole spine radiography (20). The maximally deviated level was measured on the whole spine anterior-posterior (AP) radiograph, and the horizontal distance from the central sacral vertical line (CSVL) was measured to assess trunk shift (CSVL-max) (Fig. 1). In addition, the scoliotic angle was measured between the superior endplate of S1 and the maximally tilted vertebra; the horizontal distance between the C7 plumb line and CSVL was measured (CSVL-C7) (Fig. 1). The sagittal vertical axis (SVA), lumbar curvature between T12 and S1 (LL), and pelvic parameters (pelvic incidence, Pl; sacral slope, SS; pelvic tilt, PT) were measured on the whole spine lateral radiographs (Fig. 1). Measurements were performed with the tools in the picture archiving and communication systems (Marosis, version 5483, Infinitt Healthcare, Seoul, Korea), which ran in a Microsoft Windows environment (Microsoft Corp., Redmond, WA, USA) $(16,21)$. Each measurement was performed by 2 researchers who were blinded to the patients' information.

\section{Surgical Methods}

The surgical method used had been previously described in the literature $(12,14)$. Briefly, the percutaneous endoscopic interlaminar approach and discectomy (PEID) was preferred for all patients with HIVD at L5-S1 and the percutaneous endoscopic transforaminal approach and discectomy (PETD) was selected for HIVD at L4-5 or above; the interlaminar approach was selected for 2 patients with HIVD at L4-5 due to high-grade inferior migration of the disc $(12,15)$. All operations were performed by one senior surgeon who had performed more than 700 endoscopic discectomies over the preceding 8 years $(12,15,20)$. After the induction of general anesthesia, intraoperative spontaneous electromyographic (EMG) monitoring was applied. For PETD, a line located in the middle of the disc space 
Fig. 1. Radiological measurement.

Maximum deviated vertebra was determined in the whole-spine anterior-posterior (AP) radiograph and the horizontal distance from the center of the vertebra to the central sacral vertical line (CSVL) was measured to assess trunk shift (CSVL-max). Sciatic scoliosis was measured with Cobb's angle between the superior endplate of $S 1$ and the maximally tilted vertebra. In addition, the distance between $C 7$ plumb and CSVL was measured (CSVL-C7).

Sagittal vertical axis (SVA) was measured as the horizontal distance from $C 7$ plumb to the posterior-superior corner of $S 1$, and lumbar curvature (LL) was measured with Cobb's angle between the superior endplate of T12 and $S 1$ in a whole-spine lateral radiograph. Pelvic parameters (pelvic incidence, PI; sacral slope, $S S$; pelvic tilt, PT) were measured using the measurement tools included in the picture archiving and communication systems, which used the same formula as the previous suggestion.
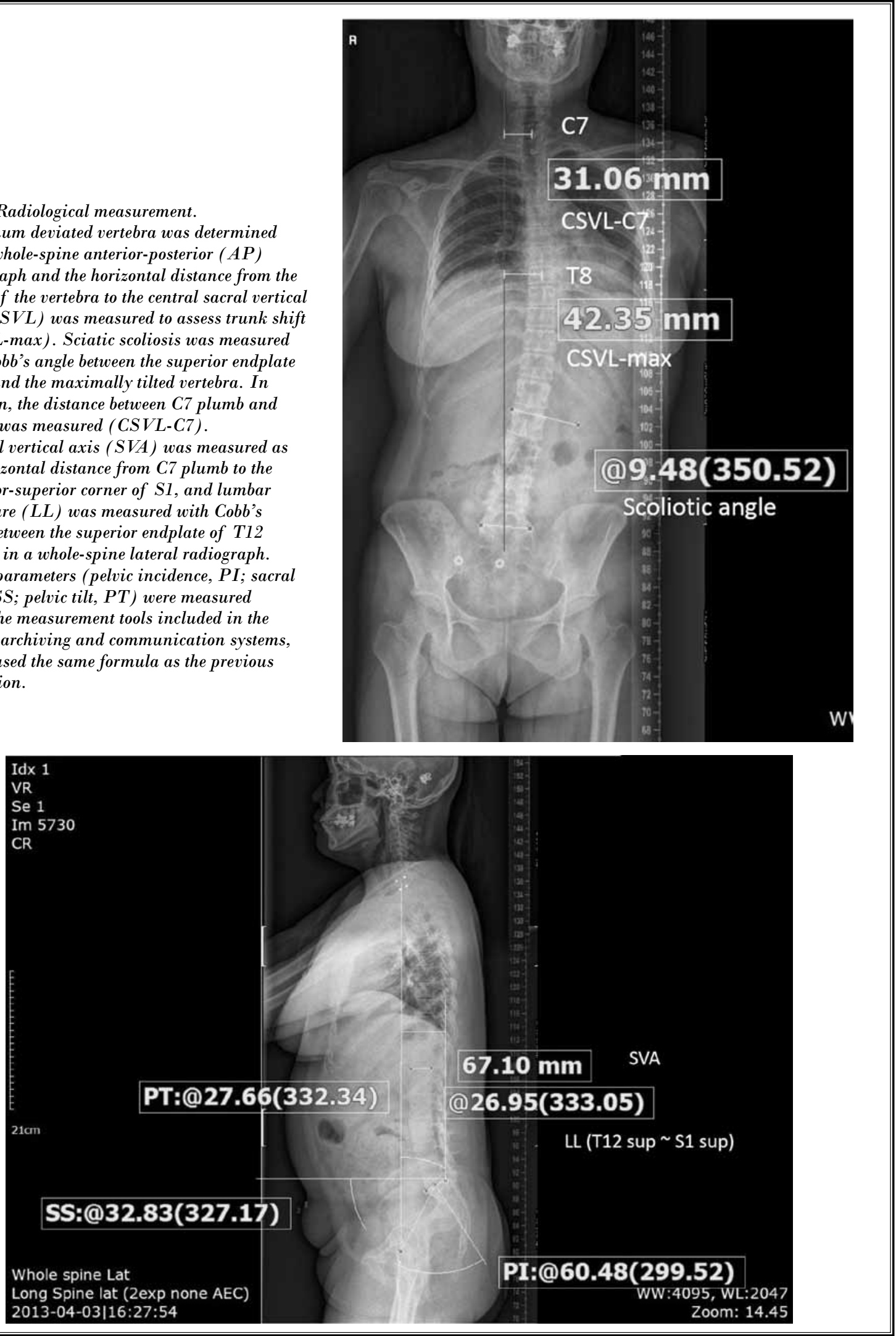
parallel with the superior endplate was marked with an AP intraoperative fluoroscopic image. The point that crossed the posterior margin of the facet joint on the lateral fluoroscopic image was selected as the entry point. A long 18-gauge needle was inserted into the neural foramen, and a guide wire was introduced after removing the stylet. The obturator was inserted over the dilator along the guide wire after making a skin incision and a working channel with a beveled opening. An endoscope (Vertebris system; Richard Wolf, Knittlingen, Germany) was then introduced into the neural foramen after removing the obturator. The ruptured disc was removed with an endoscopic instrument.

For PEID, a skin incision ( $8 \mathrm{~mm}$ ) was made midway between the medial margin of the facet joint and the spinous process in the craniocaudal center of the interlaminar window $(14,22,23)$. In the case of a highly migrated disc herniation, an oblique trajectory was chosen according to the direction and extent of the migrated disc fragment (15). A working channel with a beveled opening was inserted over the dilator, and an endoscope was introduced after removing the obturator. Under direct visualization, the ligamentum flavum was removed with scissors, and the ruptured disc was visualized after gentle retraction of the neural tissue with an obturator, which was rotated 180 degrees to allow the opening of the working channel to face the ruptured disc. The ruptured disc was removed through the opening on the beveled side of the working channel. After finishing the PEID or PETD, the working channel and endoscope were withdrawn, and the skin was closed with 3-0 nylon sutures.

\section{Statistical Analysis}

The following factors were considered for the analysis: age, gender, straight leg raise test (SLR), K-ODI, VAS-back/-leg, duration of symptoms ( $<6$ months vs. $\geq 6$ months), characteristics of HIVD (level, type [contained vs. protrusion/extrusion vs. sequestration], migration [yes vs. no], high canal compromise (24) [yes vs. no]) and radiological parameters (SVA, LL, pelvic parameters (25) [pelvic incidence, pelvic tilt, and sacral slope], scoliosis angle, CSVL-C7, and CSVL-max). Agreement between the 2 reviewers was assessed using the concordance correlation coefficients (CCC) and limits of agreement, in which the correlations among multiple observations per image were considered $(26,27)$. The analysis was performed in 2 steps: first, to identify risk factors for trunk list, and second, to describe the outcomes of trunk list after PELD.
First, the characteristics of patients with trunk list were compared with patients without trunk list using the Wilcoxon-rank sum test for continuous values and $\chi 2$-test for non-continuous values. Logistic regression analysis was utilized to identify factors for trunk list. The assumption of linearity for a continuous factor was examined using restricted cubic splines. Factors with $P$-values less than 0.2 from univariable analysis were put into multivariable analysis and the stepwise logistic regression analysis was utilized to control multi-colinearity.

Second, the outcomes of patients with trunk list after PELD were analyzed. The primary end-point was reached when trunk list was less than $10 \mathrm{~mm}$ on the follow-up whole spine AP radiograph images (Fig. 1). Because the primary end-point was measured at predetermined times (one, 3, 6, and 12 months after the operation), the event was not directly observed but was regarded to take place within the same time interval. This type of survival data is called "interval-censored survival data." To address the interval-censored data, the expectation-maximization and iterative convex minorant (EM-ICM) algorithm was used to estimate survival proportions $(28,29)$. The proportional hazard model for interval censored data was applied to verify predictors. The hazard ratio and $95 \%$ confidence interval were estimated with the ICM algorithm and bootstrap estimation using 1000 bootstrap resamples, respectively (30). The proportion hazard assumption and linearity were checked by log-log survival curves: The continuous variables were categorized by tertile points, and the log-log survival curves over these categories were analyzed. We conservatively chose the significance level as a $P$-value less than 0.05 in the univariable analysis. The significant factors in the univariable analysis were considered for the multivariable analysis. In addition to the factors used in the first analysis, the operation method and surgical outcome (favorable [excellent and good in McNab criteria] vs. unfavorable [fair and poor]) were considered for this analysis (31). Statistical analyses were performed using SAS version 9.3 (SAS Institute, Cary, NC, USA) and R software version 3.0.3 (www.r-project.org) with the intcox library.

\section{Results}

The characteristics of the patients are described in Table 1. In measuring CSVL-max, the maximally deviated vertebra was distributed as follows: T2, 1; T3, 2; T4, 2; T6, 2; T8, 1; T9, 4; T10, 1; T11, 5; T12, 5; and L1, 2 patients. Average values for each measurement were used 
Table 1. Characteristics of patients.

\begin{tabular}{|c|c|c|c|c|c|}
\hline \multirow{3}{*}{ Factors } & \multirow{3}{*}{ All } & \multicolumn{2}{|c|}{ CSVL-max } & \multicolumn{2}{|c|}{ Postoperative CSVL-max } \\
\hline & & $<10 \mathrm{~mm}$ & $\geq 10 \mathrm{~mm}$ & $<10 \mathrm{~mm}$ & $\geq 10 \mathrm{~mm}$ \\
\hline & & $(\mathrm{n}=135)$ & $(\mathrm{n}=29)$ & $(\mathrm{n}=15)$ & $(n=14)$ \\
\hline Female & 81 & 62 & 19 & 12 & 7 \\
\hline $\begin{array}{l}\text { Age, } \\
\text { Median [min, max] }\end{array}$ & $\begin{array}{c}41.0 \\
{[17.0,59.0]}\end{array}$ & $\begin{array}{c}41.0 \\
{[17.0,59.0]}\end{array}$ & $\begin{array}{c}39.0 \\
{[17.0,55.0]}\end{array}$ & $\begin{array}{c}35.0 \\
{[17.0,51.0]}\end{array}$ & $\begin{array}{c}41.0 \\
{[19.0,55.0]}\end{array}$ \\
\hline Duration of symptom & $\begin{array}{c}3.0 \\
{[1.0,48.0]}\end{array}$ & $\begin{array}{c}2.0 \\
{[1.0,48.0]}\end{array}$ & $\begin{array}{c}4.0 \\
{[1.0,12.0]}\end{array}$ & $\begin{array}{c}3.0 \\
{[1.0,12.0]}\end{array}$ & $\begin{array}{c}4.5 \\
{[1.0,12.0]}\end{array}$ \\
\hline Straight leg raising test & $\begin{array}{c}80.0 \\
{[10.0,90.0]}\end{array}$ & $\begin{array}{c}80.0 \\
{[10.0,90.0]}\end{array}$ & $\begin{array}{c}50.0 \\
{[10.0,80.0]}\end{array}$ & $\begin{array}{c}45.0 \\
{[10.0,80.0]}\end{array}$ & $\begin{array}{c}55.0 \\
{[10.0,80.0]}\end{array}$ \\
\hline Side, right : left & 79:85 & $63: 72$ & 16:13 & 11:4 & $5: 9$ \\
\hline Direction of trunk list, contralateral: ipsilateral & & & $22: 07$ & $12: 3$ & $10: 4$ \\
\hline $\begin{array}{l}\text { Type of disc (contained: protrusion/extrusion/ } \\
\text { sequestration) }\end{array}$ & $36: 128$ & $31: 104$ & $5: 24$ & $3: 12$ & 2:12 \\
\hline Migration (yes:no) & $84: 80$ & $71: 64$ & 13:16 & $5: 10$ & 8:06 \\
\hline High canal compromise (yes:no) & $32: 132$ & $23: 112$ & $9: 20$ & $4: 11$ & $5: 9$ \\
\hline Level (L4-5:L5-S1:others) & $89: 64: 11$ & $65: 59: 11$ & 24:05:00 & $12: 3$ & $12: 2$ \\
\hline K-ODI & $\begin{array}{c}19.0 \\
{[1.0,43.0]}\end{array}$ & $\begin{array}{c}18.0 \\
{[1.0,40.0]}\end{array}$ & $\begin{array}{c}19.0 \\
{[6.0,43.0]}\end{array}$ & $\begin{array}{c}25.0 \\
{[6.0,43.0]}\end{array}$ & $\begin{array}{c}19.0 \\
{[6.0,43.0]}\end{array}$ \\
\hline VAS-leg & $\begin{array}{c}6.5 \\
{[0.0,12.0]}\end{array}$ & $\begin{array}{c}6.0 \\
{[0.0,12.0]}\end{array}$ & $\begin{array}{c}7.0 \\
{[2.0,10.0]}\end{array}$ & $\begin{array}{c}7.0 \\
{[2.0,10.0]}\end{array}$ & $\begin{array}{c}6.5 \\
{[4.0,10.0]}\end{array}$ \\
\hline VAS-back & $\begin{array}{c}5.0 \\
{[0.0,12.0]}\end{array}$ & $\begin{array}{c}5.0 \\
{[0.0,12.0]}\end{array}$ & $\begin{array}{c}5.5 \\
{[0.0,10.0]}\end{array}$ & $\begin{array}{c}5.0 \\
{[0.0,8.0]}\end{array}$ & $\begin{array}{c}7.0 \\
{[0.0,10.0]}\end{array}$ \\
\hline Pelvic incidence & $\begin{array}{c}44.9 \\
{[27.4,72.4]} \\
\end{array}$ & $\begin{array}{c}44.9 \\
{[27.4,72.4]} \\
\end{array}$ & $\begin{array}{c}44.7 \\
{[34.9,68.6]} \\
\end{array}$ & $\begin{array}{c}48.2 \\
{[35.4,68.6]} \\
\end{array}$ & $\begin{array}{c}40.5 \\
{[34.9,52.4]} \\
\end{array}$ \\
\hline Pelvic tilt & $\begin{array}{c}15.4 \\
{[0.3,31.7]}\end{array}$ & $\begin{array}{c}15.0 \\
{[0.3,31.7]}\end{array}$ & $\begin{array}{c}17.2 \\
{[7.0,30.0]}\end{array}$ & $\begin{array}{c}19.2 \\
{[11.1,30.0]}\end{array}$ & $\begin{array}{c}16.0 \\
{[7.0,25.5]}\end{array}$ \\
\hline Sacral slope & $\begin{array}{c}28.9 \\
{[10.6,58.8]}\end{array}$ & $\begin{array}{c}29.9 \\
{[10.6,58.8]}\end{array}$ & $\begin{array}{c}25.3 \\
{[13.1,48.7]}\end{array}$ & $\begin{array}{c}28.3 \\
{[16.4,48.7]}\end{array}$ & $\begin{array}{c}24.7 \\
{[13.1,39.2]}\end{array}$ \\
\hline lumbar lordosis & $\begin{array}{c}-41.1 \\
{[-73.2,12.1]}\end{array}$ & $\begin{array}{c}-42.5 \\
{[-73.2,12.1]}\end{array}$ & $\begin{array}{c}-33.1 \\
{[-68.4,-9.9]}\end{array}$ & $\begin{array}{c}-33.8 \\
{[-68.4,-9.9]}\end{array}$ & $\begin{array}{c}-32.7 \\
{[-56.5,-11.7]}\end{array}$ \\
\hline SVA & $\begin{array}{c}17.4 \\
{[-59.7,246.9]} \\
\end{array}$ & $\begin{array}{c}14.1 \\
{[-59.7,246.9]} \\
\end{array}$ & $\begin{array}{c}30.8 \\
{[-27.6,159.2]}\end{array}$ & $\begin{array}{c}26.1 \\
{[-15.7,159.2]} \\
\end{array}$ & $\begin{array}{c}40.5 \\
{[-27.6,104.6]}\end{array}$ \\
\hline Sciatic scoliosis & & & $\begin{array}{c}10.5 \\
{[4.0,16.7]}\end{array}$ & $\begin{array}{c}9.3 \\
{[4.0,15.5]}\end{array}$ & $\begin{array}{c}11.7 \\
{[5.9,16.7]}\end{array}$ \\
\hline CSVL-C7 & & & $\begin{array}{c}22.5 \\
{[3.5,52.0]}\end{array}$ & $\begin{array}{c}19.8 \\
{[3.5,31.1]}\end{array}$ & $\begin{array}{c}34.3 \\
{[16.8,52.0]}\end{array}$ \\
\hline CSVL-max & & & $\begin{array}{c}27.8 \\
{[16.1,54.5]} \\
\end{array}$ & $\begin{array}{c}21.9 \\
{[16.1,44.2]}\end{array}$ & $\begin{array}{c}36.5 \\
{[17.7,54.5]} \\
\end{array}$ \\
\hline Operation (TF:IL) & $63: 101$ & $42: 93$ & $21: 8$ & $9: 6$ & $12: 2$ \\
\hline Outcome (favorable:poor) & & & $24: 5$ & $13: 2$ & $11: 3$ \\
\hline
\end{tabular}

Abbreviations: K-ODI, Korean version of Oswestry Disability Index; VAS-leg/-back, visual analogue pain score in back and arm; SVA, sagittal vertical axis; CSVL-C7, horizontal distance from the center of C7 to CSVL; CSVL-max, horizontal distance between maximally deviated vertebra to CSVL; TF, percutaneous transforaminal endoscopic disectomy; IL, percutaneous interlaminar endoscopic discectomy 
Table. 2 Risk factors for trunk list.

\begin{tabular}{|c|c|c|c|c|}
\hline & Unadjusted OR [95\% CI] & $P$-value & Adjusted OR & $P$-value \\
\hline Gender $($ ref $=$ men $)$ & $2.24[0.97,5.17]$ & 0.06 & $4.23[1.49,12.27]$ & 0.01 \\
\hline Age & $0.96[0.92,0.99]$ & 0.07 & & \\
\hline SLR & $0.96[0.94,0.97]$ & $<0.01$ & $0.94[0.92,0.97]$ & $<0.01$ \\
\hline Duration of symptom $(\mathrm{ref}=\geq 6 \mathrm{mo})$ & $1.61[1.24,2.09]$ & $<0.01$ & & \\
\hline K-ODI & & 0.36 & & \\
\hline Leg-VAS & $1.14[0.95,1.36]$ & 0.19 & & \\
\hline Trunk-VAS & & 0.77 & & \\
\hline \multicolumn{5}{|l|}{ Type of disc (ref = contained $)$} \\
\hline Protrusion/extrusion & $1.88[0.65,5.38]$ & 0.24 & & \\
\hline Sequestration & $0.22[0.02,2.01]$ & 0.18 & & \\
\hline \multicolumn{5}{|l|}{ Migration (ref = inferior) } \\
\hline No & $1.27[0.56,2.90]$ & 0.57 & & \\
\hline Superior & $0.51[0.06,4.35]$ & 0.54 & & \\
\hline High canal compromise $($ ref $=$ no $)$ & $2.19[0.89,5.42]$ & 0.09 & & \\
\hline \multicolumn{5}{|l|}{ Level (ref = L5-S1) } \\
\hline L4-L5 & $4.0[1.5,11.1]$ & 0.01 & $5.6[1.8,16.7]$ & $<0.01$ \\
\hline Others & $8.6[0.43,100]$ & 0.16 & $5.3[0.20,100]$ & 0.32 \\
\hline Pelvic incidence & & 0.37 & & \\
\hline Pelvic tilt & $1.05[0.99,1.11]$ & 0.06 & & \\
\hline Sacral slope & $0.93[0.88,0.99]$ & 0.03 & $0.92[0.87,0.99]$ & 0.02 \\
\hline Lumbar lordosis & $1.04[1.01,1.08]$ & 0.04 & & \\
\hline Sagittal vertical axis & $1.01[1.00,1.01]$ & 0.12 & & \\
\hline
\end{tabular}

for the analysis, and CCC and the limit of agreement are described in Supplemental Table $1(26,27)$. Trunk list was observed in 29/164 (17.7\%) patients (M:F = 10:19; mean age, $37.1 \pm 11.24$; range, $17-55$ ). Among them, the trunk was shifted to the contralateral side of the disc herniation in 22/29 (76\%) patients. The following factors were considered for multivariate analysis: gender, disc levels, age, duration of symptoms, SLR, SS, and LL. The characteristics of HIVD were not correlated with trunk list (Table 2). In the multivariable analysis, women experienced trunk list $(P<0.01$; OR $4.28 ; 95 \% \mathrm{Cl}, 1.49-$ 12.3) more frequently than men, and HIVD at L4-5 was significantly associated with trunk list (OR 5.6; $95 \% \mathrm{Cl}$, 1.8 - 16.7) compared with HIVD at L5-S1 (Table 2). SLR and SS were also associated with trunk list.

After PELD, trunk list was normalized $(<10 \mathrm{~mm})$ in $15(52 \%)$ patients during the 12 months after the operation, and the median deviation of $21.9 \mathrm{~mm}$ (range, 16.1 - 44.2) decreased to $6.8 \mathrm{~mm}$ (range, $3.0-9.5$ ). However, trunk shift also decreased from $36.5 \mathrm{~mm}(17.7-54.5)$ to $16.2 \mathrm{~mm}(10.6-26.9)$ in patients without normalization of trunk list. The median scoliosis angle decreased from $9.3^{\circ}$ (range, $4.0-15.5$ ) to $4.0^{\circ}$ (range, $2.6-7.5$ ) in patients with normalized trunk list. The median scoliosis angle decreased from $11.7^{\circ}$ (range, 5.9 - 16.7) to $7.2^{\circ}$ (range, 3.7 - 11.1) in patients without normalization (Table 1). The cumulative incidence rates for normalization at one, 3, 6, and 12 months were $18.8 \%, 38.8 \%$, $57.41 \%$, and $68 \%$, respectively (Fig. 2). The median time for recovery of trunk list was between 3 and 6 months. Large pelvic incidence, small CSVL-max, small CSVL-C7, and small scoliosis angle were associated with normalization of trunk list in the univariable analysis (Table 3). None of the factors in the multivariable model were significant because there was strong co-linearity among them.

\section{Discussion}

In the present study, trunk list was observed in $18 \%$ of the patients who underwent PELD. The risk factors for trunk list were female gender, L4-5 HIVD, limitation of SLR, and low SS. After PELD, trunk list was improved 
Fig. 2. Recovery from trunk list. The cumulative incidence rates for normalization at one, 3,6 , and 12 months were $18.8 \%, 38.8 \%, 57.41 \%$ and $68 \%$, respectively. The median time for recovery of trunk list was between 3 and 6 months.

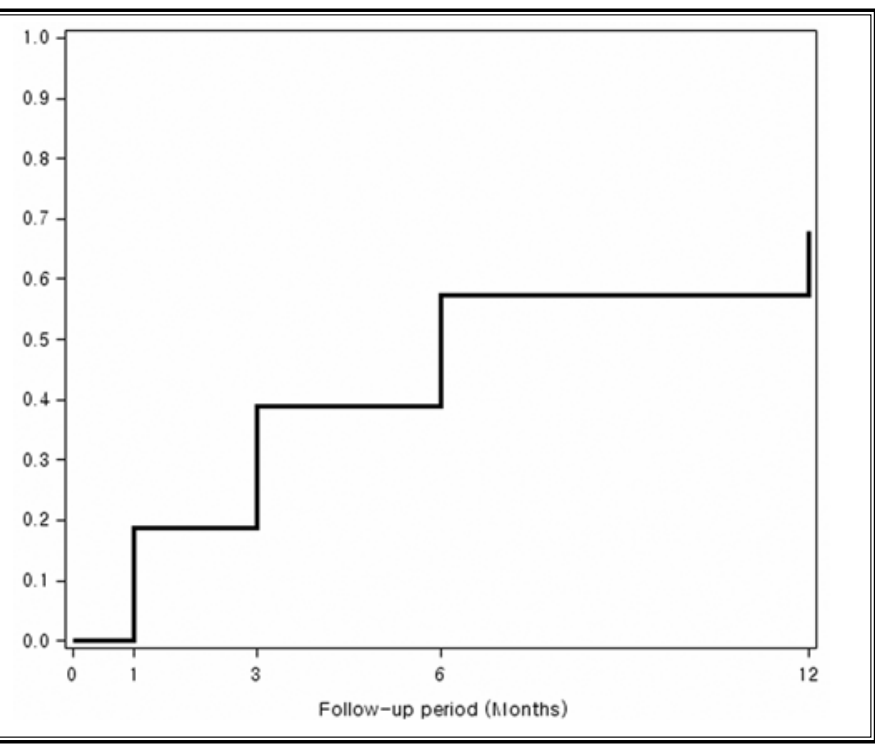

in more than $50 \%$ of patients in the $3-6$ months after surgery. The trunk shift improved by approximately 20 $\mathrm{mm}$, even in patients with persistent trunk list. Interestingly, the surgical outcome did not influence the degree of recovery from trunk list.

\section{Measuring Sciatic Trunk List}

The radiologic features of sciatic trunk list were different from idiopathic scoliosis. Sciatic scoliosis exhibited a short lumbosacral curve accompanied by a long thoracic or thoracolumbar curve toward the opposite side and a relatively straight sagittal profile (11). More than $80 \%$ of the patients showed deviation away from the painful side $(1,2,11)$.

To quantify trunk list, the Cobb's angle between L1 and L5 (S1) was used to define sciatic scoliosis $(1,4)$, but the curvature may not reflect changes in the thoracic spine $(1,4,32)$. Considering the appearance of the patients who sat, stood, and walked with a laterally shifted trunk $(2,9)$, we used CSVL-max to assess trunk shift rather than using the scoliosis angle between $L 1$ to L5. The radiological criteria that are commonly used to define trunk list by the distance of trunk shift have not been clarified. A recent report showed that trunk shift was more than $20 \mathrm{~mm}$ in $89 \%$ of patients who underwent open discectomy and trunk shift was $9 \mathrm{~mm}$ (range $5-17$ ) at 2.5 years postoperatively (11). The horizontal distance of T12 from the CSVL was measured, and it decreased from $15.0 \pm 5.9 \mathrm{~mm}$ to $7.3 \pm 5.7 \mathrm{~mm}$ with exercise therapy (9). There are 2 papers describing trunk shift by inspection of appearance $(8,17)$. Waddle et al
Table 3. Prognostic factors for recovery of trunk list.

\begin{tabular}{|c|c|}
\hline & $\begin{array}{c}\text { Unadjusted HR } \\
{[95 \% \text { CI] }}\end{array}$ \\
\hline Gender $[\mathrm{ref}=\mathrm{men}]$ & $1.83[0.49,2.6 \times 108]$ \\
\hline Age & $1.02[0.98,1.08]$ \\
\hline Straight leg raising test & $0.99[0.96,1.02]$ \\
\hline Duration of symptom [ref $=\geq 6 \mathrm{mo}]$ & $0.86[0.52,1.02]$ \\
\hline Direction of deviation $[\mathrm{ref}=$ contralateral $]$ & $1.01[0.28,3.12]$ \\
\hline K-ODI & $1.06[0.98,1.15]$ \\
\hline Leg-VAS & $1.1[0.84,1.63]$ \\
\hline Trunk-VAS & $0.84[0.65,1.01]$ \\
\hline Disc type $[$ ref = contained $]$ & $0.76[0.19,7.3 \times 107]$ \\
\hline Migration $[\mathrm{ref}=\mathrm{no}]$ & $0.7[0.18,1.99]$ \\
\hline High canal compromise $[\mathrm{ref}=$ yes $]$ & $0.43[0.05,1.07]$ \\
\hline Surgical level [ref = L4-5] & $1.16[0,7.46]$ \\
\hline Pelvic incidence ${ }^{*}$ & $1.11[1,1.28]$ \\
\hline Pelvic tilt & $1.07[0.96,1.21]$ \\
\hline Sacral slope & $1.04[0.95,1.13]$ \\
\hline Lumbar lordosis & $0.99[0.94,1.04]$ \\
\hline Sagittal vertical axis & $1.01[0.99,1.02]$ \\
\hline Sciatic scoliosis ${ }^{*}$ & $0.81[0.57,0.99]$ \\
\hline CSVL-C7* & $0.96[0.92,0.99]$ \\
\hline CSVL-max ${ }^{*}$ & $0.94[0.87,0.98]$ \\
\hline Operation [ref = interlaminar approach] & $0.5[0.12,1.49]$ \\
\hline
\end{tabular}

*: $P<0.05$ 
(17) quantified trunk list based on a gross inspection, and a deviation of more than $10 \mathrm{~mm}$ from the midline was categorized as trunk list. McLean et al (8) inspected the appearance of trunk list and categorized trunk list as a greater than $10 \mathrm{~mm}$ deviation from the midline and recommended the use of a plumb line for measurement. We adopted those criteria in our radiological classification of trunk list.

\section{Natural Course of Trunk List}

Sciatic trunk list was not uncommon and was observed in $40 / 446(9 \%)$ of the patients who needed lumbar discectomy (1) in the literature and in 29/164 (18\%) of the patients in the present study. It was hypothesized that the trunk was tilted laterally in response to irritation of the nerve root or hyperactivity of the paraspinal muscles $(1-4,6,7,10,32,33)$. Previously, although sciatic trunk list was not structural, bracing was prescribed as treatment (2). We now understand that trunk list is reversible by reducing pain without the use of braces $(1,2,4,7,10)$. This improvement might be enhanced with exercise therapy $(7,9)$. With non-surgical treatment, trunk list resolved in $50 \%$ of patients without McKenzie exercises but resolved in $91 \%$ of patients with McKenzie exercises (statistically not significant) over the span of 3 months (7). The Harrison mirror image exercise (lateral translation lumbar traction exercise) improved trunk shift by approximately $7 \mathrm{~mm}$ during 2.5 months (9). However, surgical treatment is required for medically intractable patients. Theoretically, trunk list due to HIVD could be improved once the irritation of the nerve root is relieved $(1,2,4,7,10)$. After open discectomy, sciatic scoliosis completely disappeared in $45 \%(18 / 40)$ of the patients by a mean of 7.5 months and in $69 \%$ (31/45) of the patients by 7 days $(1,4)$. Although the surgical outcome is not substantially different between open discectomy and PELD $(13,14)$, the outcome of trunk list may be different because the injury to the musculature and ligament may be different from open discectomy (5). The present study showed that trunk list was normalized in more than half of the patients within 6 months, which seemed to be poorer than the previous results. However, the criterion of trunk list was different from previous reports $(1,4)$, and direct comparison was not possible; the present study did not include patients with scoliosis of more than $4^{\circ}$ or less than $10 \mathrm{~mm}$ deviation. In the previous literature, the deviation was ranged from 1 to 64 $\mathrm{mm}$ (mean 16.7) for patients with $>4^{\circ}$ sciatic scoliosis (4).

\section{Factors Related to Trunk List}

In addition to the horizontal distance from the midline, we measured LL and pelvic parameters to assess the changes in the lumbar spine and pelvis because it has been suggested that the response to tonic contraction of the surrounding lumbo-pelvic muscles may be related to trunk list (5). The appearance of patients with trunk list was characterized as lateral and forward trunk list and lumbar hypolordosis (2). Although the present study compared values among patients with HIVD, patients with trunk list had lower SS and LL and higher SVA than did patients without trunk list (Table 2). It seemed that radiological changes also reflected the appearance of patients. Patients with trunk list also had more limitation in SLR, which suggested that the irritation of the nerve root may be more severe in patients with trunk list than in patients without it. Trunk list was more frequently observed in patients with HIVD at L4-5 in the present study (24/29) and in previous reports (29/40 and 34/45) $(1,4)$. Because L4-5 was not confined to the pelvic cavity, it may be more vulnerable to trunk list compared with L5-S1. These results suggest that an unbalanced lumbo-pelvic muscular response to irritation of the nerve root may result in trunk list $(1-4,6,7,10,32,33)$. Although the female gender was associated with trunk list in the present study, sciatic scoliosis was more frequently observed in men in previous reports $(1,4)$.

The pathophysiology of trunk list may be complex, and the compression of a nerve root by herniated disc material may not be a sole causative factor for sciatic trunk list, even in patients with HIVD. Trunk list was observed even in patients without leg pain; irritation of the nerve root was not a necessary condition $(1,2,7,9)$. In addition, the location or degree of nerve root compression was not correlated with the amount of trunk deviation $(1,4)$. There was a weak correlation between the amount of trunk list and the low-back pain/disability index $(7,34)$. Moreover, the disability index was not different between patients with resolution of trunk list and patients without resolution of trunk list (7). Those findings were also observed in the present study, and the failure to resolve trunk list even after successful relief of pain supports the argument that the compression of the nerve root by disc material may not be the sole cause for trunk list. The prognostic factors for recovery of trunk list may be evident with analysis of a greater number of patients. 


\section{Limitations of the Present Study}

Selection bias should be considered in interpreting these results. The analysis was performed with surgically treated patients, and comparison with a normal population is relevant to characterize patients with trunk list. In addition, the surgeries were performed in a tertiary-referral hospital, in which the incidence of trunk list may be higher. Nearly all patients in the present study underwent several spinal epidural injections and these interventions might bias the outcome $(13,18)$. Moreover, the small size of the study may result in statistical error.

Nonetheless, the present study showed that the characteristic appearance of patients with trunk list was reflected in the radiological measurements, and that radiological change could be reversed. This information may be valuable for clinicians and patients in consultation.

\section{Conclusion}

Trunk list was observed in $18 \%$ of the patients at the time of surgery. Female gender and L4-5 disc herniation were risk factors for trunk list. Trunk list was reversible in more than $50 \%$ of patients within 6 months of PELD. A larger study is required to identify prognostic factors for the recovery from trunk list.

\section{Supplemental table 1.}

\begin{tabular}{|l|c|c|c|c|c|}
\hline \multirow{2}{*}{ Variable } & \multicolumn{5}{|c|}{ Bland-Altman method } \\
\cline { 2 - 5 } & $\begin{array}{c}\text { \# of paired } \\
\text { patients }\end{array}$ & $\begin{array}{c}\text { \# of paired } \\
\text { observations }\end{array}$ & Bias & Limit of agreement & CCC [95\% CI] \\
\hline PI & 157 & 385 & 5.10 & {$[-6.15,16.34]$} & $0.697[0.628,0.754]$ \\
\hline PT & 157 & 385 & -0.30 & {$[-4.85,4.25]$} & $0.948[0.933,0.959]$ \\
\hline SS & 157 & 385 & 5.46 & {$[-4.62,15.54]$} & $0.615[0.541,0.679]$ \\
\hline LL & 157 & 386 & -4.77 & {$[-18.05,8.51]$} & $0.782[0.729,0.825]$ \\
\hline SVA & 153 & 375 & 0.47 & {$[-9.51,10.44]$} & $0.992[0.990,0.993]$ \\
\hline Max tilt & 29 & 107 & 0.82 & {$[-1.91,3.55]$} & $0.818[0.695,0.895]$ \\
\hline CSVL-C7 & 29 & 107 & -0.36 & {$[-4.51,3.79]$} & $0.972[0.949,0.984]$ \\
\hline CSVL-max & 29 & 107 & -0.98 & {$[-7.51,5.56]$} & $0.939[0.891,0.966]$ \\
\hline
\end{tabular}

\section{References}

1. Matsui H, Ohmori K, Kanamori M, Ishihara $H$, Tsuji $H$. Significance of sciatic scoliotic list in operated patients with lumbar disc herniation. Spine (Phila $\mathrm{Pa}$ 1976) 1998; 23:338-342.

2. Kleinberg S. Sciatic scoliosis. Am J Surg 1950; 80:332-337.

3. Krishnan KM, Newey ML. Lumbar scoliosis associated with a disc herniation in an adult. Rheumatology (Oxford) 2001; 40:1427-1428.

4. Suk KS, Lee HM, Moon SH, Kim NH. Lumbosacral scoliotic list by lumbar disc herniation. Spine (Phila Pa 1976) 2001; 26:667-671.

5. Endo K, Suzuki H, Tanaka $H$, Kang $Y$, Yamamoto K. Sagittal spinal alignment in patients with lumbar disc herniation. Eur Spine ] 2010; 19:435-438.
6. Lorio MP, Bernstein AJ, Simmons EH. Sciatic spinal deformity-lumbosacral list: An "unusual" presentation with review of the literature. J Spinal Disord 1995; 8:201-205.

7. Gillan MG, Ross JC, McLean IP, Porter RW. The natural history of trunk list, its associated disability and the influence of McKenzie management. Eur Spine J 1998; 7:480-483.

8. McLean IP, Gillan MG, Ross JC, Aspden RM, Porter RW. A comparison of methods for measuring trunk list. A simple plumbline is the best. Spine (Phila Pa 1976) 1996; 21:1667-1670.

9. Harrison DE, Cailliet R, Betz JW, Harrison DD, Colloca CJ, Haas JW, Janik TJ, Holland B. A non-randomized clinical control trial of Harrison mirror image methods for correcting trunk list (lateral translations of the thoracic cage) in patients with chronic low back pain. Eur Spine ] 2005; 14:155-162.

10. Spanos GP. Sciatic scoliosis, its natural history and the ability of the Mckenzie management to influence it. Stud Health Technol Inform 2002; 91:332-335.

11. Zhu Z, Zhao Q, Wang B, Yu Y, Qian B, Ding Y, Qiu Y. Scoliotic posture as the initial symptom in adolescents with lumbar disc herniation: Its curve pattern and natural history after lumbar discectomy. BMC Musculoskelet Disord 2011; 12:216.

12. Kim $\mathrm{CH}$, Chung $\mathrm{CK}$, Sohn S, Lee $\mathrm{S}$, Park SB. The surgical outcome and the surgical strategy of percutaneous endoscopic discectomy for recurrent disk 
herniation. J Spinal Disord Tech 2014; 27:415-422.

13. Kim CH, Chung CK, Park CS, Choi B, Kim MJ, Park BJ. Reoperation rate after surgery for lumbar herniated intervertebral disc disease: Nationwide cohort study. Spine (Phila Pa 1976) 2013; 38:581-590.

14. Ruetten S, Komp M, Merk H, Godolias G. Full-endoscopic interlaminar and transforaminal lumbar discectomy versus conventional microsurgical technique: A prospective, randomized, controlled study. Spine (Phila Pa 1976) 2008; 33:931-939.

15. Kim CH, Chung CK, Woo JW. Surgical outcome of percutaneous endoscopic interlaminar lumbar discectomy for highly migrated disc herniation. J Spinal Disord Tech 2012. Oct 15. [Epub ahead of print]

16. Kim $\mathrm{CH}$, Chung $\mathrm{CK}$, Hong HS, Kim EH, Kim MJ, Park BJ. Validation of a simple computerized tool for measuring spinal and pelvic parameters.] Neurosurg Spine 2012; 16:154-162.

17. Waddell G, Main C), Morris EW, Venner RM, Rae PS, Sharmy SH, Galloway H. Normality and reliability in the clinical assessment of backache. Br Med J (Clin Res Ed) 1982; 284:1519-1523.

18. Park $\mathrm{CH}$, Lee $\mathrm{SH}$, Park HS. Lumbar retrodiscal versus post-ganglionic transforaminal epidural steroid injection for the treatment of lumbar intervertebral disc herniations. Pain Physician 2011; 14:353-360.

19. Kim DY, Lee SH, Lee HY, Lee HJ, Chang SB, Chung SK, Kim HJ. Validation of the Korean version of the oswestry disability index. Spine (Phila Pa 1976) 2005; 30:E123-E127.

20. Kim $\mathrm{CH}$, Shin $\mathrm{KH}$, Chung $\mathrm{CK}$, Park SB, $\mathrm{Kim} \mathrm{JH}$. Changes in cervical sagittal alignment after single-level posterior percutaneous endoscopic cervical diskectomy. Global Spine J 2015; 5:31-38.

21. Barrey C, Jund J, Noseda O, Roussouly $P$. Sagittal balance of the pelvis-spine complex and lumbar degenerative diseases. A comparative study about 85 cases. Eur Spine J 2007; 16:1459-1467.

22. Choi G, Lee SH, Raiturker PP, Lee $S$, Chae YS. Percutaneous endoscopic interlaminar discectomy for intracanalicular disc herniations at $\mathrm{L}_{5}$-S $\mathrm{S}$ using a rigid working channel endoscope. Neurosurgery 2006; 58:ONS59-68.

23. Kim $\mathrm{CH}$, Chung $\mathrm{CK}$, Jahng $\mathrm{TA}$, Yang $\mathrm{HJ}$, Son YJ. Surgical outcome of percutaneous endoscopic interlaminar lumbar diskectomy for recurrent disk herniation after open diskectomy. J Spinal Disord Tech 2012; 25:E125-E133.

24. Lee SH, Kang BU, Ahn Y, Choi G, Choi YG, Ahn KU, Shin SW, Kang HY. Operative failure of percutaneous endoscopic lumbar discectomy: A radiologic analysis of 55 cases. Spine (Phila Pa 1976) 2006; 31:E285-E290.

25. Legaye J, Duval-Beaupere G, Hecquet J, Marty C. Pelvic incidence: A fundamental pelvic parameter for three-dimensional regulation of spinal sagittal curves. Eur Spine ] 1998; 7:99-103.

26. Carrasco JL, Phillips BR, Puig-Martinez J, King TS, Chinchilli VM. Estimation of the concordance correlation coefficient for repeated measures using SAS and R. Comput Methods Programs Biomed 2013; 109:293-304.

27. Carrasco JL, Jover L. Estimating the generalized concordance correlation coefficient through variance components. Biometrics 2003; 59:849-858.

28. Wellner JA, Zhan YH. A hybrid algorithm for computation of the nonparametric maximum likelihood estimator from censored data. J Am Stat Assoc 1997; 92:945-959.

29. Huang J, Lee C, Yu Q. A generalized logrank test for interval-censored failure time data via multiple imputation. Stat Med 2008; 27:3217-3226.

30. Pan W. Extending the iterative convex minorant algorithm to the Cox model for interval-censored data. J Comput Graph Stat 1999; 8:109-120.

31. Macnab I. Negative disc exploration. An analysis of the causes of nerve-root involvement in sixty-eight patients. J Bone Joint Surg Am 1971; 53:891-903.

32. Hirayama J, Yamagata M, Ogata S, Shimizu K, Ikeda Y, Takahashi K. Relationship between low-back pain, muscle spasm and pressure pain thresholds in patients with lumbar disc herniation. Eur Spine J 2006; 15:41-47.

33. Takahashi K, Shima I, Porter RW. Nerve root pressure in lumbar disc herniation. Spine (Phila Pa 1976) 1999; 24:2003-2006.

34. Arangio GA, Hartzell SM, Reed JF. Significance of lumbosacral list and lowback pain. A controlled radiographic study. Spine (Phila Pa 1976) 1990; 15:208-210. 